\title{
Bone morphogenetic protein 9 serves a protective role in response to ischemic-reperfusion in the brain by promoting ERK activation
}

\author{
YINLING FENG and YIDA HU
}

\begin{abstract}
Department of Neurology, The First Affiliated Hospital of Chongqing Medical University, Chongqing 400016, P.R. China
\end{abstract}
Received January 21, 2017; Accepted September 11, 2017

DOI: $10.3892 / \mathrm{mmr} .2017 .8253$

\begin{abstract}
The aim of the present study was to investigate the expression and function mechanism of bone morphogenetic protein 9 (BMP9) in cerebral ischemia-reperfusion (I/R) injuries in vivo and in vitro. A total of 40 Sprague-Dawley rats were randomly divided into four groups $(n=10)$ : i) Normal control; ii) sham surgery group, the procedure without occlusion; iii) I/R group, right middle cerebral artery occlusion (MCAO) followed by reperfusion; and iv) adenoviral vector (Ad)-BMP9 + I/R group, Ad-BMP9 intracerebroventricular injection was performed 2 days prior to MCAO. Neurological deficit score and infarct volume were measured at $24 \mathrm{~h}$ following reperfusion. To further test the mechanism of BMP9, astrocytes were isolated and treated with Ad-BMP9, Ad-BMP9 + extracellular signal-regulated kinase (ERK) inhibitor PD098059, Ad-BMP9 + c-Jun N-terminal kinase inhibitor SP600125 and Ad-BMP9 + p38 inhibitor SB203580 for $24 \mathrm{~h}$, followed by undergoing oxygen-glucose deprivation and reoxygenation (OGD/R) treatment. Cell viability and death were assessed by 3-(4,5-dimethylthiazol-2yl)-5-(3-carboxymethoxyphenyl)(4-sulfophenyl)-2H-tetrazolium and lactate dehydrogenase release, respectively. Gene expression was determined by quantitative polymerase chain reaction and western blotting. BMP9 was identified to be upregulated at mRNA and protein levels in cerebral I/R animal and cell models. BMP9 pretreatment significantly reduced the neurological score and infarct volume compared with I/R rats. In astrocytes, overexpression of BMP9 significantly decreased cell death and improved cell viability, an effect which may be mediated by the ERK signaling pathway, as ERK was activated by BMP9 and the use of PD098059 partially reversed the protective effect of BMP9. Pretreatment with BMP-9 may be a promising treatment option for prevention of cerebral I/R injuries.
\end{abstract}

Correspondence to: Dr Yida Hu, Department of Neurology, The First Affiliated Hospital of Chongqing Medical University, 1 You Yi Road, Chongqing 400016, P.R. China

E-mail: wanghdhdh@163.com

Key words: bone morphogenetic protein 9, ischemia-reperfusion injury, apoptosis, extracellular signal-regulated kinases

\section{Introduction}

Stroke is the second most prevalent cause of mortality worldwide and $\sim 8$ million succumb to it annually (1). Ischemic stroke accounts for $\sim 80 \%$ of strokes, and results from the occlusion of a major intracerebral artery by a thrombus or embolism (2). Re-canalization of occluded cerebral blood vessels is the effective approach to restore the cerebral flow and minimize deleterious effects of ischemia on neurons (3). However, several studies have indicated that ischemic-reperfusion (I/R) usually induces reactive oxygen species overproduction, which are highly deleterious and result in neuronal cell injury or death $(4,5)$. Therefore, it has become important to explore ways to prevent cerebral $\mathrm{I} / \mathrm{R}$ injuries.

Bone morphogenetic proteins (BMPs) are members of the transforming growth factor- $\beta$ (TGF- $\beta$ ) superfamily that were originally identified as factors that induce the formation of bone and cartilage (6). However, recent studies indicate BMPs are also expressed in the central nervous system and involved in the growth, differentiation and apoptosis of neuronal cells under normal and pathological conditions, including cerebral I/R (7-9). BMP family members include the BMP-2/4 group (BMP2 and BMP4), osteogenic protein-1 group (BMP5, BMP6, BMP7 and BMP8) and BMP-9/10 group (BMP9 and BMP10) (10). Of these, certain members have been demonstrated to exert protective roles for cerebral I/R injuries. For example, Xu et al (11) demonstrated that the administration of rhBMP7 via a femoral vein injection $30 \mathrm{~min}$ prior to reperfusion significantly increases neurological function, decreases brain water content and pathological and morphological damage, and may be associated with reduced nuclear factor- $\kappa \mathrm{B}$ activity. Using the same procedure, Pei et al (12) also demonstrated that BMP-7 significantly improves neurological deficiency and reduces the infarct volume via attenuating oxidative stress and inhibiting neuronal apoptosis following cerebral ischemia. By in vitro and vivo experimentation, Wang et al (13) demonstrated that BMP6 treatment may reduce cerebral ischemia/reperfusion injury by alleviating neuronal apoptosis, with decreased immunoreactivity, enzymatic activity of caspase-3, and density of terminal deoxynucleotidyl transferase dUTP nick end labeling-positive cells in the ischemic cortex. However, there have been no studies, to the best of the authors' knowledge, investigating the role of BMP9, a newly identified factor for developing basal forebrain cholinergic neurons (14), in cerebral I/R injuries. 
The aim of the present study was to investigate whether BMP9 has a protective role in preventing focal cerebral (IR) injuries and their underlying mechanisms. The results may provide insight into the underlying functional role of BMP9 in the nervous system lesion and repair.

\section{Materials and methods}

Animals and experimental groups. Adult male SpragueDawley rats ( $\mathrm{n}=40 ; 280-330 \mathrm{~g}$; aged between 16 and 17 weeks) were obtained from the Experiment Animal Center of Chongqing Medical University. They were housed in rooms with controlled temperature $\left(22-25^{\circ} \mathrm{C}\right)$, humidity $(50-60 \%)$ and a 12-h light/dark cycle, with free access to food and water. All rats were randomly divided into four groups $(\mathrm{n}=10)$ : i) Normal control; ii) sham surgery group; iii) I/R group; and iv) Ad-BMP9 + I/R group. All animal experiments were approved by the Institutional Animal Care and Use Committee of Chongqing Medical University and conducted in accordance with the principles and procedures outlined in the National Institutes of Health Guide for the Care and Use of Laboratory Animals (https://grants.nih.gov/grants/olaw/ Guide-for-the-Care-and-use-of-laboratory-animals.pdf).

Construction of focal cerebral $I / R$ model. The transient focal cerebral $I / R$ injury model was induced via the right middle cerebral artery occlusion (MCAO) as described by Longa et al (15). Briefly, rats were anesthetized by intraperitoneal injection of $350 \mathrm{mg} / \mathrm{kg}$ chloral hydrate (Sigma-Aldrich; Merck KGaA, Darmstadt, Germany) following an overnight fast and placed in a supine position. A midline neck incision was made to expose the right common carotid artery, internal carotid artery (ICA) and external carotid artery (ECA). A 4-0 monofilament nylon suture (Ethicon, Inc., Osaka, Japan) with a rounded tip was inserted from the lumen of the ECA to that of the right ICA until a resistance was felt to occlude the origin of the right middle cerebral artery. Throughout the procedure, body temperature was maintained within the physiological range by a thermostatically controlled infrared lamp. Reperfusion was performed $2 \mathrm{~h}$ following MCAO via thread withdrawal. Sham-surgery rats underwent the same vessel exposure, however without MCAO.

Adenoviral vector (Ad)-BMP9 expression vector construction. The adenoviral vectors carrying BMP9 and/or GFP were obtained as a gift from Molecular Oncology Laboratory, the University of Chicago Medical Center (Chicago, IL, USA). They were constructed using the AdEasy System (Invitrogen; Thermo Fisher Scientific, Inc., Waltham, MA, USA) according to the manufacturer's protocol. The recombinant adenoviruses were further packaged and amplified in 293 cells (ATCC, Manassas, VA, USA) followed by storage at $-80^{\circ} \mathrm{C}(16)$.

Intracerebroventricular infusion of Ad-BMP9. Ad-BMP9 was intracerebroventricularly injected into the rat brain 2 days prior to MCAO. In brief, rats were anesthetized with $10 \%$ chloral hydrate $(3 \mathrm{ml} / \mathrm{kg})$ and placed in a stereotaxic frame. Ad-BMP9 $(0.1 \mathrm{ml})$ was injected into the right lateral ventricle via a $1 \mathrm{~mm}$ burr hole drilled $1.5 \mathrm{~mm}$ caudal to the bregma and $2 \mathrm{~mm}$ lateral to the midline. The needle was kept in the ventricle for $10 \mathrm{~min}$, and then withdrawn. Following the closure of the wound, the rats were returned to their cages and fed as normal.

Evaluation of neurological deficits. Neurological deficits were evaluated $24 \mathrm{~h}$ following reperfusion according to the method of Longa et al (15). Neurological findings were scored on a 5-point scale: 0 points, no deficit; 1 point, failure to extend left forepaw; 2 points, circling to the left; 3 points, paresis to the left; 4 points, no walking spontaneously with depressed level of consciousness. The cerebral I/R model was considered to be successfully constructed with a neurological deficits score $>3$.

Estimation of cerebral infarct volume. The cerebral infarct volume was determined by 2,3,5-triphenyltetrazolium chloride (TTC) staining (17). Briefly, the rats were deeply anesthetized with chloral hydrate (10\% chloral hydrate; $3 \mathrm{ml} / \mathrm{kg}$ ) $24 \mathrm{~h}$ following reperfusion and then decapitated, following which the brains were rapidly removed and frozen at $-20^{\circ} \mathrm{C}$ for $20 \mathrm{~min}$. Brain tissue was sliced into five $2 \mathrm{~mm}$ sections and stained with $2 \%$ TTC solution for $30 \mathrm{~min}$ at $37^{\circ} \mathrm{C}$ in the dark followed by overnight immersion in $4 \%$ paraformaldehyde. The tissue slices were digitally photographed and the volume of the infarction (indicated as a white color) was analyzed and expressed as percentage relative to the total brain volume using the ImageJ software (version 1.37; National Institutes of Health, Bethesda, MD, USA).

Isolation and culture of astrocytes. Astrocytes were dissociated from eight neonatal Sprague-Dawley rats (Experiment Animal Center of Chongqing Medical University) (18). Briefly, the head of neonatal rats was cut off following anesthesia (10\% chloral hydrate; $3 \mathrm{ml} / \mathrm{kg}$ ) and the brains were removed from the skulls. Following careful removal of the meninges and pia mater, the brain tissues were cut into pieces by scissors and digested with trypsin for $10 \mathrm{~min}$ at $37^{\circ} \mathrm{C}$, then plated on poly-L-lysine-coated culture dishes with Dulbecco's modified Eagle's medium (DMEM) (Gibco; Thermo Fisher Scientific, Inc.) supplemented with $10 \%$ fetal bovine serum (Gibco; Thermo Fisher Scientific, Inc.) in a humidified atmosphere of $5 \% \mathrm{CO}_{2}$ at $37^{\circ} \mathrm{C}$. The culture medium was changed every 2 days. When the primary cells reached $80-95 \%$ confluence, the cells were detached by trypsinization, centrifuged at $300 \mathrm{x} \mathrm{g}$ for $5 \mathrm{~min}$ at room temperature, and replaced. The subsequent passage cells were used for the following experiment when $85-100 \%$ confluence was achieved.

Simulation of oxygen-glucose deprivation and reoxygenation model. An oxygen-glucose deprivation and reoxygenation (OGD/R) model was constructed as previously described (19). Having being washed twice, astrocytes were immersed in glucose-free DMEM and placed in an incubator with a premixed gas $\left(1 \% \mathrm{O}_{2}, 95 \% \mathrm{~N}_{2}\right.$ and $\left.5 \% \mathrm{CO}_{2}\right)$ for $4 \mathrm{~h}$. Then, cells were maintained in normal DMEM (including $5.6 \mathrm{mmol} / 1$ glucose) and transferred to a $5 \% \mathrm{CO}_{2}$ incubator at $37^{\circ} \mathrm{C}$ for $24 \mathrm{~h}$. For the non-OGD/R group, cultures were incubated in normal DMEM and placed in $5 \% \mathrm{CO}_{2}$ in air at $37^{\circ} \mathrm{C}$ for $28 \mathrm{~h}$. Astrocytes were also treated with Ad-GFP, Ad-BMP9, Ad-BMP9 + extracellular signal-regulated kinases (ERK) inhibitor PD098059 (20 $\mu \mathrm{M}$; Sigma-Aldrich; Merck KGaA), Ad-BMP9 + c-Jun N-terminal kinase (JNK) inhibitor 
SP600125 (20 $\mu \mathrm{m}$; Cell Signaling Technology, Inc., Danvers, MA, USA), Ad-BMP9 + p38 inhibitor SB203580 (20 $\mu \mathrm{m}$; Cell Signaling Technology, Inc.), PD098059 (20 $\mu \mathrm{m})$, SP600125 $(20 \mu \mathrm{m})$, and SB203580 $(20 \mu \mathrm{m})$ for $24 \mathrm{~h}$ and then subjected to OGD/R treatment.

Cell viability analysis. Cell viability was assessed by 3-(4,5-dimethylthiazol-2yl)-5-(3-carboxymethoxyphenyl)(4-sulfophenyl)-2H-tetrazolium (MTS) method. Astrocytes were cultured in 96-well plates and MTS solution added. Following $3 \mathrm{~h}$ in the dark, cells were measured on a microplate reader at a wavelength of $490 \mathrm{~nm}$ (Bio-Rad Laboratories, Inc., Hercules, CA, USA).

Cell death analysis. A lactate dehydrogenase release assay was used to indirectly evaluate cytotoxicity (cell death) of astrocytes following OGD/R, which was performed using a commercially available kit (Nanjing Jiancheng Bioengineering Institute, Nanjing, China). Absorbance was detected at a wavelength of $450 \mathrm{~nm}$ on microplate reader (Bio-Rad Laboratories, Inc.).

Reverse transcription-quantitative polymerase chain reaction $(R T-q P C R)$. RT-qPCR was used to investigate the mRNA expression of BMP9, ERK, P38 and JNK. Total RNA was isolated from the brain tissues or astrocytes using RNAiso Plus (Takara Biotechnology Co., Ltd., Dalian, China) according to the manufacturer's protocol. cDNA was synthesized with PrimeScript RT reagent kit (Takara Biotechnology Co., Ltd.). The PCR primers used were: BMP9 forward, 5'-CCT TCTTTCATTCCCTCTGTGA-3' and reverse, 5'-CCCAAC CTTTTGACCCTTTTTA-3', 143 bp; ERK forward, 5'-TGA AGGATGACGACTTTGAGAA-3' and reverse, 5'-CTGTAG AACGCACCATAGAAGC-3', 217 bp; P38 forward, 5'-GAG CGTTACCAGAACCTGTCTC-3' and reverse, 5'-TGAATG ATGGACTGAAATGGTC-3', 128 bp; JNK forward, 5'-GAC ACGAAGACGAACTTGAGC-3' and reverse, 5'-CGTAAT AGGCAGGAAAGACACC-3' 126 bp; and $\beta$-actin forward, 5'-CACCCGCGAGTACAACCTTC-3' and reverse, 5'-CCC ATACCCACCATCACACC-3', 207 bp). Real-time qPCR was run on a Bio-Rad CFX-96 real-time PCR system (Bio-Rad Laboratories, Inc.) using SYBR Premix Ex Taq kit (Takara Biotechnology Co., Ltd.) at $95^{\circ} \mathrm{C}$ for $30 \mathrm{sec}$, followed by 39 cycles of $95^{\circ} \mathrm{C}$ for $5 \mathrm{sec}$, and $60^{\circ} \mathrm{C}$ for $30 \mathrm{sec}$. The gene expression levels relative to internal standard $\beta$-actin were calculated by the $2^{-\Delta \Delta C q}$ method (20).

Western blotting. Western blotting was used to investigate the protein expression of BMP9, ERK, P38 and JNK. The protein samples were extracted from brain tissues or astrocytes using RIPA lysis buffer (BioTeke Corporation, Beijing, China) and then protein levels were determined using the bichioninic acid method. A $20 \mu \mathrm{g}$ protein sample was subjected to $12 \%$ SDS-PAGE gel and transferred onto polyvinylidene fluoride membranes. Blots were blocked with $5 \%$ non-fat milk solution for $1 \mathrm{~h}$ at room temperature and then incubated overnight with anti-BMP9 (cat. no. sc514211; 1:2,000; Santa Cruz Biotechnology, Inc., Dallas, TX, USA), anti-p-ERK (cat. no. BS74621; 1:2,000; Bioworld Technology, Inc., St. Louis Park, MN, USA), anti-p-P38 (cat. no. BS6381; 1:2,000;
Bioworld Technology, Inc.), or anti-p-JNK (cat. no. Bs4763; 1:2,000; Bioworld Technology, Inc.) at $4{ }^{\circ} \mathrm{C}$ followed by incubation with horseradish peroxidase-conjugated goat anti-rabbit IgG secondary antibodies (cat. no. ZB2301; 1:3,000; Beijing Zhongshan Jinqiao Biotechnology Co., Ltd., Beijing, China) for $1 \mathrm{~h}$. The blots were visualized with the ECL system (GE Healthcare Life Sciences, Little Chalfont, UK) and the intensity of each band quantified by using the Chemi Doc XRS system.

Statistical analysis. RNA and protein assay data are presented as the mean \pm standard error of the mean, which were repeated three times. Differences between groups were analyzed using Tukey's post hoc test following one-way analysis of variance through SPSS software, version 18.0 (SPSS, Inc., Chicago, IL, USA). A t-test was used to compare the differences between two groups. $\mathrm{P}<0.05$ was considered to indicate a statistically significant difference. The area of TTC staining and cerebral infarction was photographed with a digital camera, and results analyzed using Image J software (version 1.37)

\section{Results}

Cerebral I/R induces the upregulated expression of BMP9. To investigate whether BMP9 is involved in cerebral I/R, the expression of BMP9 was first examined in cerebral I/R animal and cell models. As demonstrated in Fig. 1, BMP9 was identified to be upregulated at mRNA and protein levels in the cerebral I/R animal model triggered by MACO and the cell model following OGD/R treatment.

Effect of BMP9 overexpression on neurological function and infarction. To confirm whether the role of upregulated BMP9 is protective or deteriorative, BMP9 was overexpressed via adenovirus vector mediation followed by cerebral I/R treatment. Previous studies have suggested that cerebral I/R leads to neurological deficit and cerebral infarction $(21,22)$. Thus, the role of BMP9 overexpression on neurological function was assessed according to the score defined by Longa et al (15) and infarct volume stained by TTC. As a result, normal and sham surgery rats presented almost no neurological deficit and infarct volume, however this significantly increased in the I/R group. Furthermore, BMP9 pretreatment significantly reduced the neurological score and infarct volume compared with I/R rats (Fig. 2).

Effect of BMP9 overexpression on cell viability and death of astrocytes. In addition to the animal model, the overexpression of BMP9 on astrocytes was also analyzed. In agreement with the animal experiment, cell viability of astrocytes was significantly reduced, however the death of astrocytes was enhanced following OGD/R treatment. However, the BMP9 pretreatment significantly alleviated this condition, with the increased cell viability and lower cell death rate (Fig. 3).

Underlying alleviation mechanism of BMP9 for cerebral I/R. To further explore the mechanism of BMP9 on cerebral I/R, three signaling pathways (p38, ERK and JNK) that usually 
A

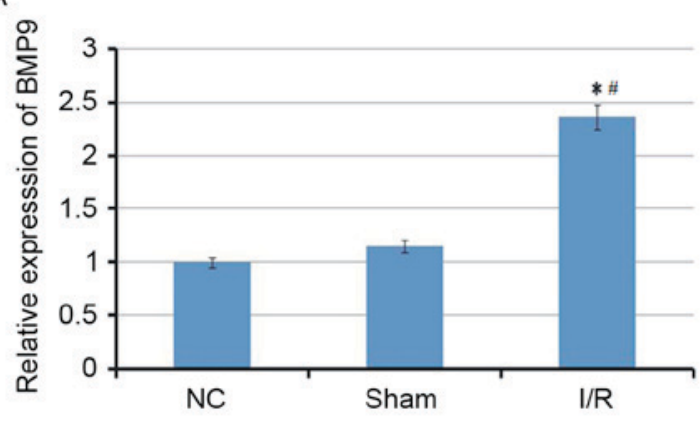

C

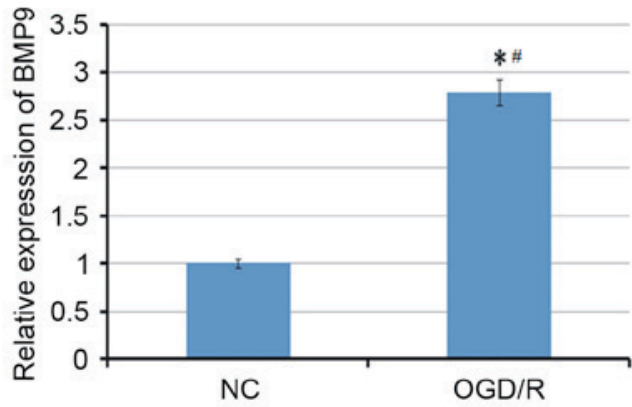

B

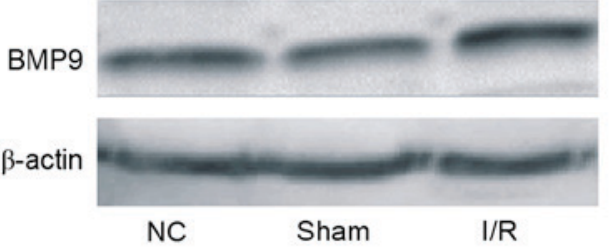

D

BMP9

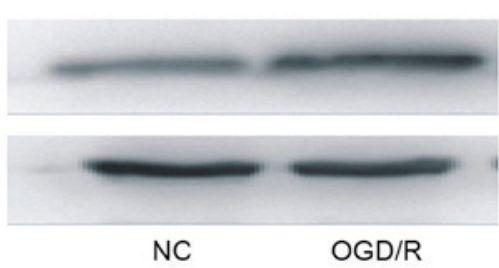

Figure 1. Expression of BMP9 in cerebral ischemia reperfusion animal and cell models. (A and B) Rat model induced via the right middle cerebral artery occlusion. (A) mRNA expression level and (B) protein expression level. (C and D) Astrocyte model induced via oxygen-glucose deprivation and reoxygenation. (C) mRNA expression level and (D) protein expression level. " $\mathrm{P}<0.05$ vs. $\mathrm{NC}$ and ${ }^{\#} \mathrm{P}<0.05$ vs. sham. BMP9, bone morphogenetic protein 9; NC, normal control group; sham, sham surgery group; I/R, cerebral ischemia reperfusion group; OGD/R, oxygen-glucose deprivation and reoxygenation group.

A

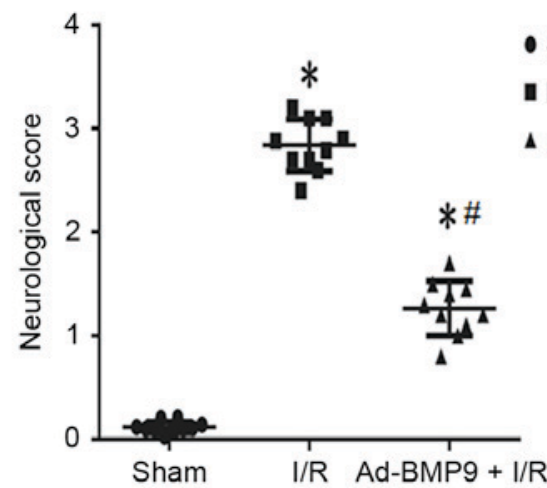

B

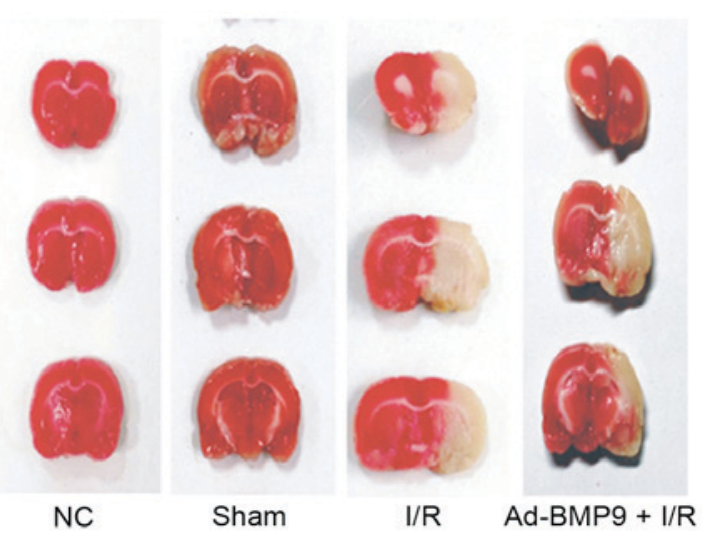

- Sham

- I/R

$\Delta$ Ad-BMP9 + I/R

C

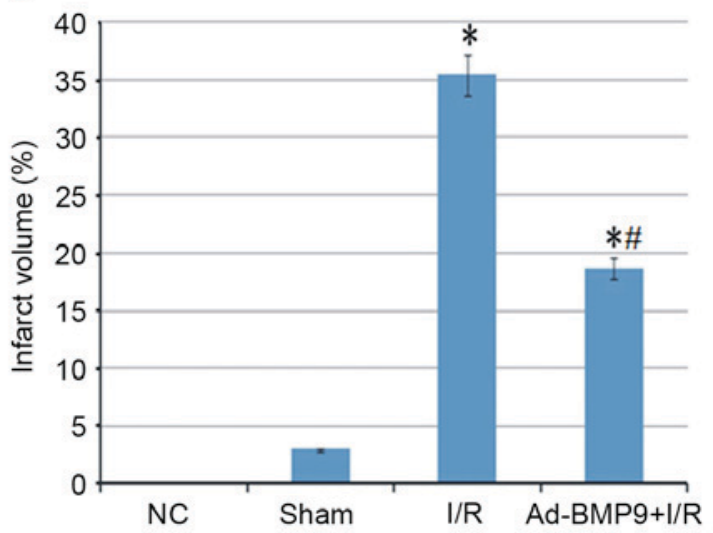

Figure 2. Effect of BMP9 overexpression on neurological function and infarction. (A) quantitative analysis of neurological score. (B) Representative TTC staining in cerebral sections. (C) Quantitative analysis of infarct volume. " $\mathrm{P}<0.05$ vs. sham and ${ }^{\#} \mathrm{P}<0.05 \mathrm{vs}$. I/R group. BMP9, bone morphogenetic protein 9 ; TTC, 2,3,5-triphenyltetrazolium chloride; sham, sham surgery group; I/R, cerebral ischemia reperfusion group; NC, normal control group; Ad-BMP9, Adenoviral vector-BMP9 expression vector group. 
A

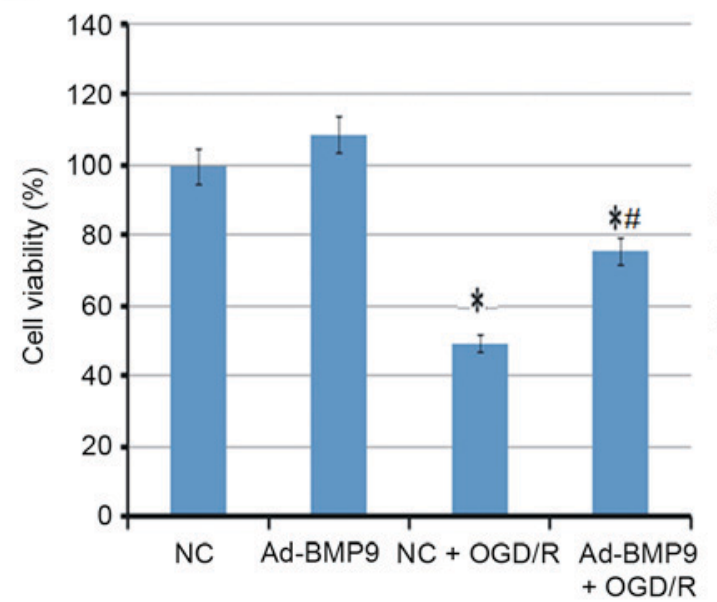

B

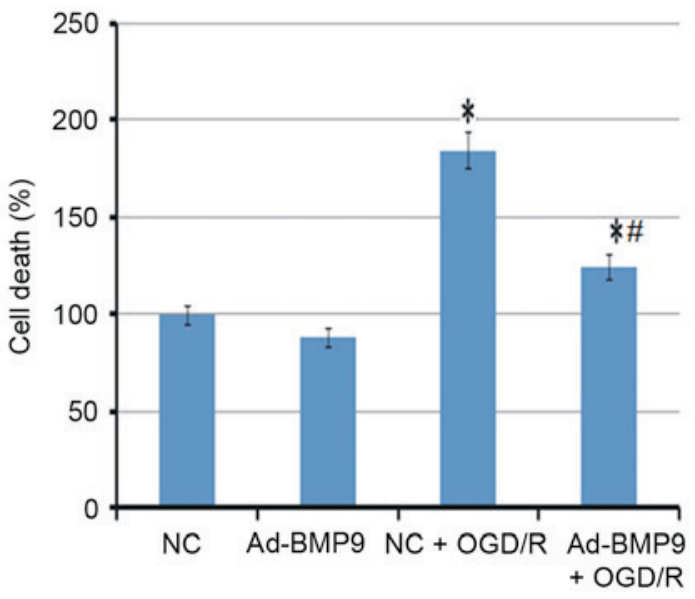

Figure 3. Effect of BMP9 overexpression on cell viability and death of astrocytes. (A) Cell viability determined by MTS. (B) Cell death determined by lactate dehydrogenase release. ${ }^{~} \mathrm{P}<0.05$ vs. $\mathrm{NC}$ and ${ }^{~} \mathrm{P}<0.05$ vs. $\mathrm{NC}+$ astrocytes model induced via OGD/R group. BMP9, bone morphogenetic protein 9; MTS, 3-(4,5-dimethylthiazol-2yl)-5-(3-carboxymethoxyphenyl)-(4-sulfophenyl)-2H-tetrazolium; NC, normal control group; OGD/R, oxygen-glucose deprivation and reoxygenation; Ad-BMP9, Adenoviral vector-BMP9 expression vector group.

A
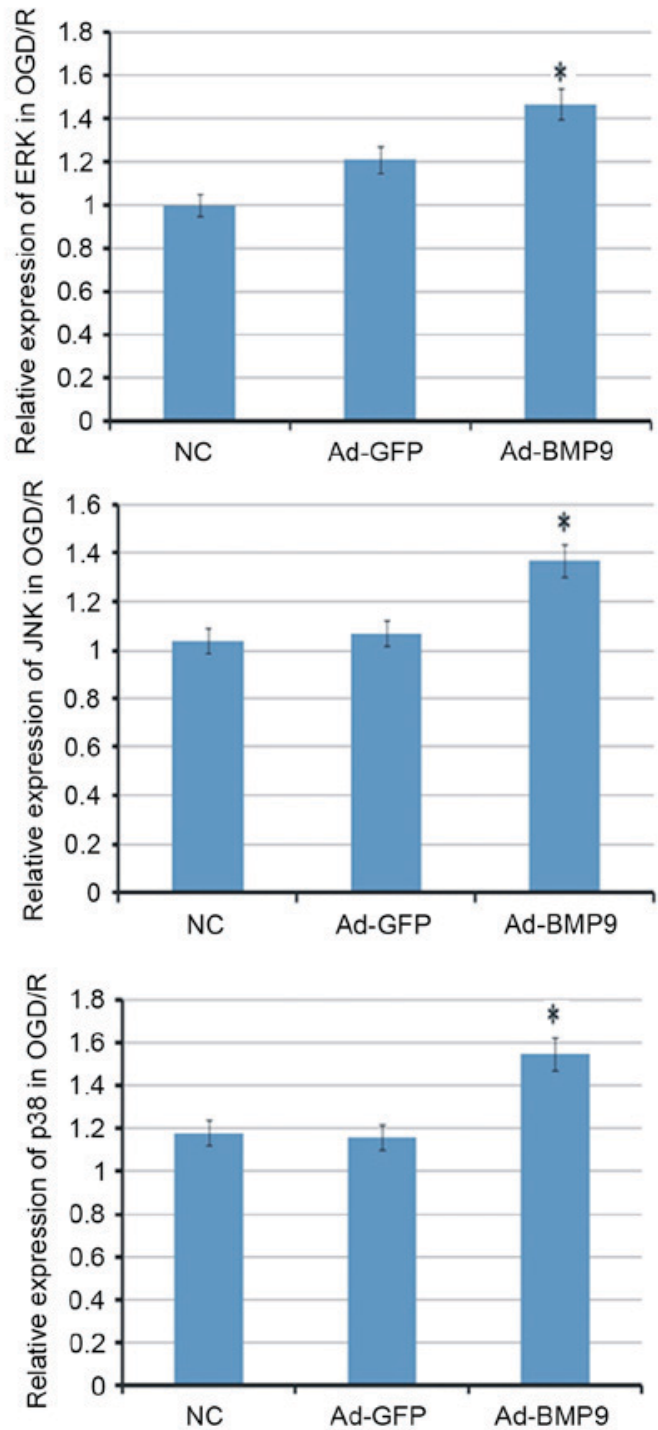

B

ERK

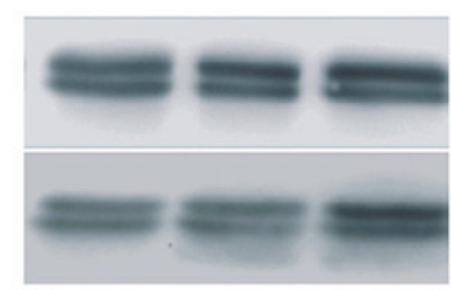

JNK

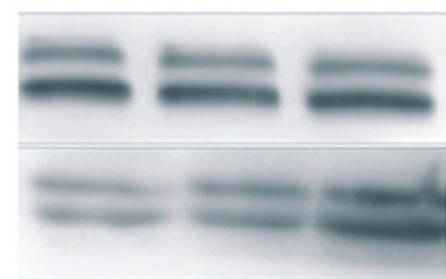

p38

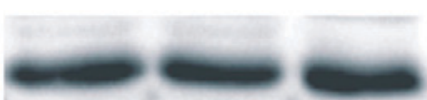

P-p38

$\beta$-actin

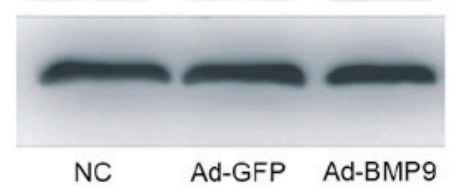

Figure 4. Effect of BMP9 overexpression on ERK, JNK and p38 in astrocytes undergoing oxygen-glucose deprivation and reoxygenation (OGD/R). (A) mRNA expression level. (B) Protein expression level. "P<0.05 vs. NC. BMP9, bone morphogenetic protein 9; ERK, extracellular signal-regulated kinase; JNK, c-Jun N-terminal kinase; NC, normal control group; p-, phospho-; Ad-BMP9, Adenoviral vector-BMP9 expression vector group. 
A

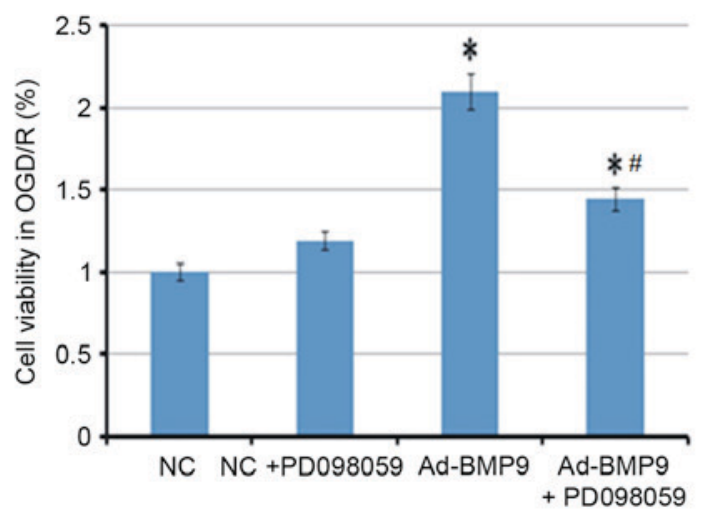

C

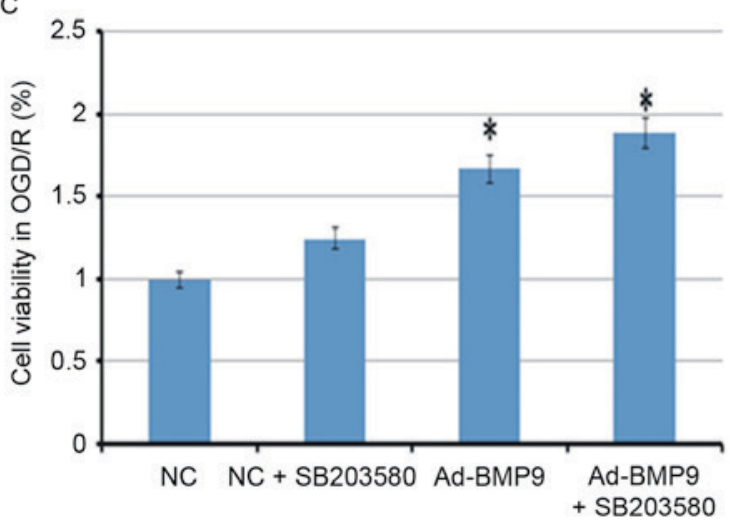

E

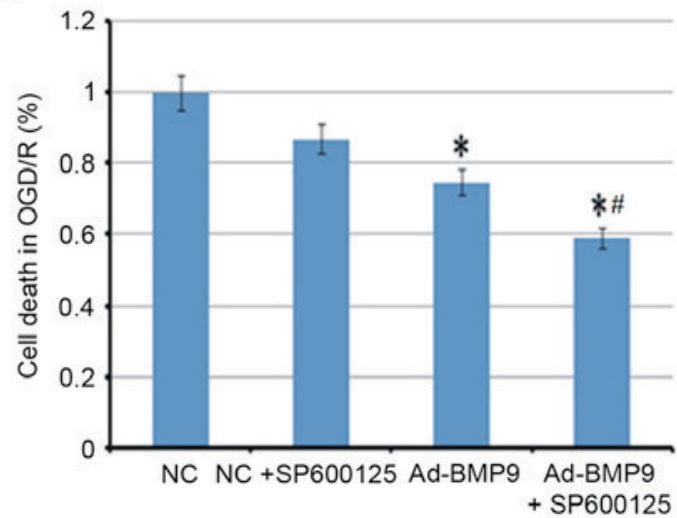

B

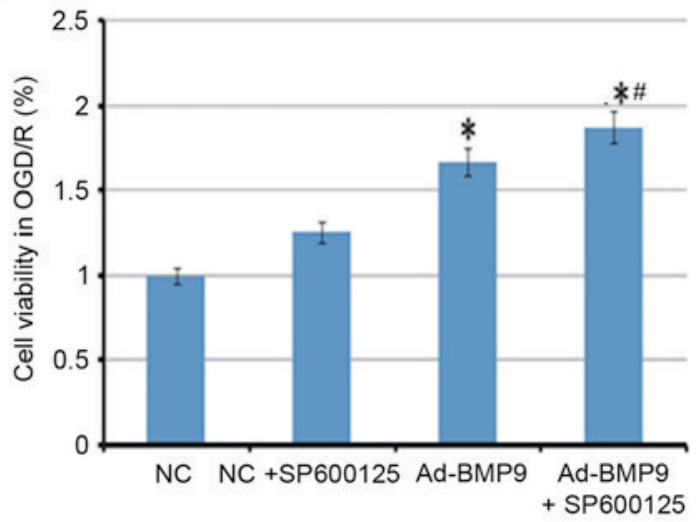

D

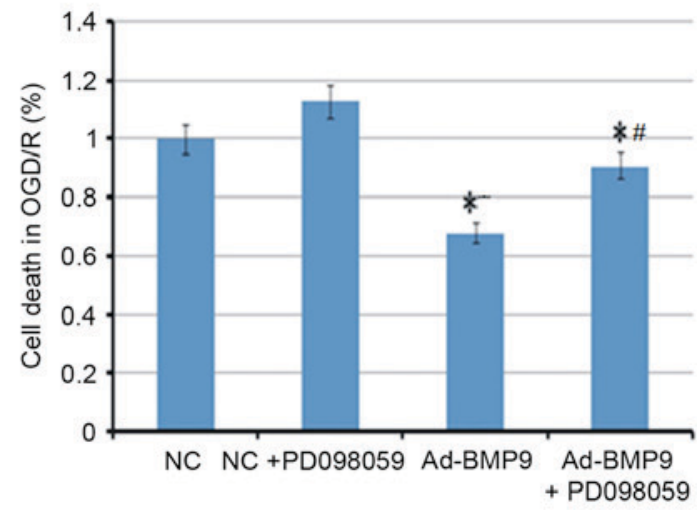

$\mathrm{F}$

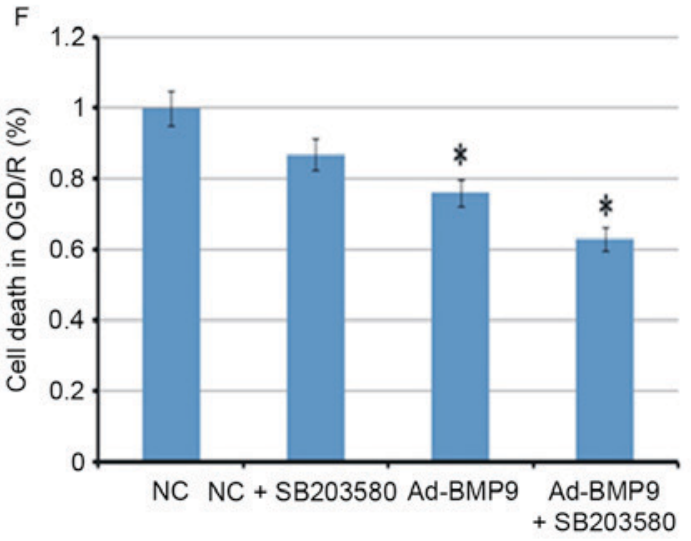

Figure 5. Inhibition of ERK, JNK and p38 on cell viability and death of astrocytes undergoing oxygen-glucose deprivation and reoxygenation (OGD/R). Cell viability determined by MTS for inhibition of (A) ERK, (B) JNK and (C) p38. Cell death determined by lactate dehydrogenase release for inhibition of (D) ERK, (E) JNK and (F) p38. "P<0.05 vs. NC and ${ }^{~} \mathrm{P}<0.05$ vs. Ad-BMP9. ERK, extracellular signal-regulated kinase; JNK, c-Jun N-terminal kinase; MTS, 3-(4,5-dimethylthiazol-2yl)-5-(3-carboxymethoxyphenyl)-(4-sulfophenyl)-2H-tetrazolium; NC, normal control group; Ad-BMP9, Adenoviral vector-BMP9 expression vector group.

serve important roles in cell survival, were determined. The results indicated that the mRNA expression levels of $\mathrm{p} 38$, ERK and JNK were all increased in OGD/R-treated astrocytes. At the protein levels, the expression of p38, ERK and JNK appeared to be similar in different groups, however, their phosphorylation levels were improved by BMP9 with different degrees (Fig. 4).

In addition, inhibitors of p38, ERK and JNK were also used to further demonstrate that the protective role of BMP9 in cerebral I/R is p38-, ERK- and JNK-mediated. Notably, only ERK inhibitor PD098059 was identified as reversing the protective role of BMP9, with the reduced cell viability and increased death of astrocytes compared with BMP9 alone, whereas the JNK inhibitor SP600125 and p38 inhibitor SB203580 further improved the protective role of BMP9, indicating the parallel mechanism with BMP9 (Fig. 5).

\section{Discussion}

Although accumulating evidence has demonstrated that BMP9 is expressed in brain neurons $(14,23,24)$, this is the first study, to the best of the authors' knowledge, to explore its functional 
mechanism in cerebral I/R injuries. The results demonstrated that BMP9 is a protective response molecule that is significantly upregulated in the cerebral I/R animal and cell models. Pretreatment with BMP9 effectively ameliorates neurological defect and brain infarct volume, which may be associated with its ability to reduce death and improve viability of astrocytes. The findings of the present study appeared to be in line with previous studies on the other members of the BMP family $(9,13,25)$.

BMP9 has been reported to exert its function on osteogenesis and the maintenance of bone cell survival by activating the downstream mitogen-activated protein kinase (MAPK) signaling pathway $(26,27)$. It is also clear that MAPK pathway members, including ERK, JNK and p38, serve important roles in neuronal cells as a response to the stress stimuli induced by I/R (28). Inhibition of ERK by U0126 or PD98059 significantly decreases the number of surviving cells in hippocampal CA1 subfield and cortex (29), whereas activation of ERK significantly ameliorates neurological deficit and histopathology alterations in rats exposed to $90 \mathrm{~min} \mathrm{MCAO}$-induced ischemia and $24 \mathrm{~h}$ reperfusion $(30,31)$. The alterations in JNK and p38 appeared to be contrary to ERK, with the inhibition of JNK and p38 attenuating infarction volume in I/R brains in vivo $(32,33)$.

To further confirm whether the protective role of BMP9 in cerebral I/R injuries is MAPK signaling pathway mediated, the expression levels of ERK, JNK and p38 were measured in addition to the use of ERK, JNK and p38 inhibitors in the OGD/R injured neurons. Although the expression of ERK, JNK and p38 appeared to be increased following BMP9 treatment, only the ERK inhibitor partially reversed the effect of BMP9 on the death and viability of astrocytes, indicating that activation of ERK may be a mechanism underlying the neuroprotective effects of BMP9.

The present study has certain limitations. First, the investigation of the neuroprotection effect of BMP9 in vivo was relatively simple. Apoptosis and proliferation of neurons and the effect of ERK inhibitor were not analyzed. Second, the neurological deficit score and infarct volume were evaluated only at $24 \mathrm{~h}$ of reperfusion and the long-term effect of BMP9 on neurological outcome remains unclear. Third, the downstream mediators of ERK were not assessed in vitro and in vivo.

In conclusion, the present study preliminarily demonstrated that pretreatment with BMP-9 may be a promising option for the prevention of cerebral $\mathrm{I} / \mathrm{R}$ injuries, and may reduce death and maintain the growth of neurons by stimulating the ERK signaling pathway.

\section{References}

1. Roger VL, Go AS, Lloyd-Jones DM, Adams RJ, Berry JD, Brown TM, Carnethon MR, Dai S, de Simone G, Ford ES, et al: Heart disease and stroke statistics-2011 update: A report from the American Heart Association. Circulation 123: e18-e209, 2011.

2. Stoll G, Kleinschnitz C and Nieswandt B: Molecular mechanisms of thrombus formation in ischemic stroke: Novel insights and targets for treatment. Blood 112: 3555-3562, 2008.

3. Deb P, Sharma S and Hassan KM: Pathophysiologic mechanisms of acute ischemic stroke: An overview with emphasis on therapeutic significance beyond thrombolysis. Pathophysiology 17: $197-218,2010$
4. Sanderson TH, Reynolds CA, Kumar R, Przyklenk K and Hüttemann M: Molecular mechanisms of ischemia-reperfusion injury in brain: Pivotal role of the mitochondrial membrane potential in reactive oxygen species generation. Mol Neurobiol 47: 9-23, 2013.

5. Wang M, Li YJ, Ding Y, Zhang HN, Sun T, Zhang K, Yang L, Guo YY, Liu SB, Zhao MG and Wu YM: Silibinin prevents autophagic cell death upon oxidative stress in cortical neurons and cerebral ischemia-reperfusion Injury. Mol Neurobiol 53: 932-943, 2016

6. Reddi AH: Regulation of cartilage and bone differentiation by bone morphogenetic proteins. Curr Opin Cell Biol 4: 850-855, 1992.

7. Liu A and Niswander LA: Bone morphogenetic protein signalling and vertebrate nervous system development. Nat Rev Neurosci 6: 945-954, 2005.

8. Chalazonitis A and Kessler JA: Pleiotropic effects of the bone morphogenetic proteins on development of the enteric nervous system. Dev Neurobiol 72: 843-856, 2012.

9. Zhang R, Pei H, Ru L, Li H and Liu G: Bone morphogenetic protein 7 upregulates the expression of nestin and glial fibrillary acidic protein in rats with cerebral ischemia-reperfusion injury. Biomed Rep 1: 895-900, 2013.

10. Miyazono K, Kamiya Y and Morikawa M: Bone morphogenetic protein receptors and signal transduction. J Biochem 147: 35-51, 2010.

11. Xu JH, Zhang TZ, Zhao YY, Wang JK and Yuan ZG: Protective effects of recombinant human bone morphogenetic protein-7 on focal cerebral ischemia-reperfusion injury. Int J Neurosci 123: 375-384, 2013.

12. Pei H, Cao D, Guo Z, Liu G, Guo Y and Lu C: Bone morphogenetic protein-7 ameliorates cerebral ischemia and reperfusion injury via inhibiting oxidative stress and neuronal apoptosis. Int J Mol Sci 14: 23441-23453, 2013.

13. Wang Y, Chang CF, Morales M, Chou J, Chen HL, Chiang YH, Lin SZ, Cadet JL, Deng X, Wang JY, et al: Bone morphogenetic protein- 6 reduces ischemia-induced brain damage in rats. Stroke 32: 2170-2178, 2001.

14. Schnitzler AC, Mellott TJ, Lopez-Coviella I, Tallini YN, Kotlikoff MI, Follettie MT and Blusztajn JK: BMP9 (bone morphogenetic protein 9) induces NGF as an autocrine/paracrine cholinergic trophic factor in developing basal forebrain neurons. J Neurosci 30: 8221-8228, 2010.

15. Longa EZ, Weinstein PR, Carlson S and Cummins R: Reversible middle cerebral artery occlusion without craniectomy in rats. Stroke 20: 84-91, 1989.

16. Zhao C, Wu N, Deng F, Zhang H, Wang N, Zhang W, Chen X, Wen S, Zhang J, Yin L, et al: Adenovirus-mediated gene transfer in mesenchymal stem cells can be significantly enhanced by the cationic polymer polybrene. PLoS One 9: e92908, 2014.

17. Bederson JB, Pitts LH, Germano SM, Nishimura MC, Davis RL and Bartkowski HM: Evaluation of 2,3,5-triphenyltetrazolium chloride as a stain for detection and quantification of experimental cerebral infarction in rats. Stroke 17: 1304-1308, 1986.

18. Chen H, Tian M, Jin L, Jia H and Jin Y: PUMA is invovled in ischemia/reperfusion-induced apoptosis of mouse cerebral astrocytes. Neuroscience 284: 824-832, 2015.

19. Dong YF, Chen ZZ, Zhao Z, Yang DD, Yan H, Ji J and Sun XL: Potential role of microRNA-7 in the anti-neuroinflammation effects of nicorandil in astrocytes induced by oxygen-glucose deprivation. J Neuroinflammation 13: 60, 2016.

20. Livak KJ and Schmittgen TD Analysis of relative gene expression data using real-time quantitative PCR and the 2(-Delta Delta C(T)) method. Methods 25: 402-408, 2001.

21. Moskowitz MA, Lo EH and Iadecola C: The science of stroke: Mechanisms in search of treatments. Neuron 67: 181-198, 2010.

22. Lo EH: Experimental models, neurovascular mechanisms and translational issues in stroke research. Br J Pharmacol 153 (Suppl 1): S396-S405, 2008.

23. López-Coviella I, Berse B, Krauss R, Thies RS and Blusztajn JK: Induction and maintenance of the neuronal cholinergic phenotype in the central nervous system by BMP-9. Science 289: 313-316, 2000

24. Lopez-Coviella I, Follettie MT, Mellott TJ, Kovacheva VP, Slack BE, Diesl V, Berse B, Thies RS and Blusztajn JK: Bone morphogenetic protein 9 induces the transcriptome of basal forebrain cholinergic neurons. Proc Natl Acad Sci USA 102: 6984-6989, 2005. 
25. Luan L, Yang X, Zhou C, Wang K and Qin L: Post-hypoxic and ischemic neuroprotection of BMP-7 in the cerebral cortex and caudate-putamen tissue of rat. Acta Histochem 117: 148-154, 2015.

26. Fong D, Bisson M, Laberge G, Mcmanus S, Grenier G, Faucheux N and Roux S: Bone morphogenetic protein-9 activates Smad and ERK pathways and supports human osteoclast function and survival in vitro. Cell Signal 25: 717-728, 2013.

27. Ye G, Li C, Xiang X, Chen C, Zhang R, Yang X, Yu X, Wang J, Wang L, Shi Q and Weng Y: Bone morphogenetic protein- 9 induces PDLSCs osteogenic differentiation through the ERK and p38 signal pathways. Int J Med Sci 11: 1065-1072, 2014.

28. Kovalska M, Kovalska L, Pavlikova M, Janickova M, Mikuskova K, Adamkov M, Kaplan P, Tatarkova Z and Lehotsky J: Intracellular signaling MAPK pathway after cerebral ischemia-reperfusion injury. Neurochem Res 37: 1568-1577, 2012.

29. Zhu YM, Wang CC, Chen L, Qian LB, Ma LL, Yu J, Zhu MH, Wen CY, Yu LN and Yan M: Both PI3K/Akt and ERK1/2 pathways participate in the protection by dexmedetomidine against transient focal cerebral ischemia/reperfusion injury in rats. Brain Res 1494: 1-8, 2013.
30. Wang PR, Wang JS, Zhang C, Song XF, Tian $N$ and Kong LY: Huang-Lian-Jie-Du-Decotion induced protective autophagy against the injury of cerebral ischemia/reperfusion via MAPK-mTOR signaling pathway. J Ethnopharmacol 149: 270-280, 2013

31. Yang S, Yuan Y, Jiao S, Luo Q and Yu J: Calcitonin gene-related peptide protects rats from cerebral ischemia/reperfusion injury via a mechanism of action in the MAPK pathway. Biomed Rep 4: 699-703, 2016.

32. Gong J, Sun F, Li Y, Zhou X, Duan Z, Duan F, Lei Z, Chen H, Qi S and Shen J: Momordica charantia polysaccharides could protect against cerebral ischemia/reperfusion injury through inhibiting oxidative stress mediated c-Jun N-terminal kinase 3 signaling pathway. Neuropharmacology 91: 123-134,2015.

33. Wang W, Tang L, Yong L and Yong W: Biochanin a protects against focal cerebral ischemia/reperfusion in rats via inhibition of p38-mediated inflammatory responses. J Neurol Sci 348 121-125, 2015.

This work is licensed under a Creative Commons Attribution-NonCommercial-NoDerivatives 4.0 International (CC BY-NC-ND 4.0) License. 